\title{
La noción de significado convencional en la interfaz semántica-pragmática
}

\author{
EDUARDO DOMINICCINI \\ Catedrático \\ Instituto Caro y Cuervo \\ Calle 10 N. 4-69, Bogotá, Colombia \\ E-mail: ledominiccini@uc.cl
}

LA NOCIÓN DE SIGNIFICADO
CONVENCIONAL EN LA INTER-
FAZ SEMÁNTICA-PRAGMÁTICA

RESUMEN: En el presente trabajo se discute la noción de "significado convencional" dentro de la interfaz semántica-pragmática. Esta noción es el punto de partida para establecer la distinción griceana entre lo dicho por el hablante y lo implicado o el contenido comunicado. En este trabajo se critica tal línea, poniendo en cuestión la utilidad de dicha noción. La discusión se divide en dos partes. La primera parte defiende que la noción de lo convencional no captura adecuadamente las propiedades de interfaz que pretenden describir y explicar quienes la adoptan. En la segunda parte se plantea que la aceptación o el rechazo con respecto a la convencionalidad como criterio de análisis del significado depende parcialmente de si podemos o no aceptar que las intuiciones sobre el contenido semántico y el enriquecimiento pragmático de las expresiones lingüisticas deben ser tenidas en cuenta en una teoria integral y adecuada del significado.

PALABRAS CLAVES: interfaz; semántica; pragmática; significado convencional; intuición.

SUMARIO: 1. Introducción. 2. Las interfaces del significado. 2.1. La interfaz semántica-pragmática (ISP). 3. La distinción entre significado convencional y no convencional en la ISP. 3.1. El problema de lo (no) convencional en la ISP. 4. Intuiciones frente a convencionalidad. 4.1. El rol de las intuiciones en la interfaz. 5. Conclusiones.
THE NOTION OF CONVENTIONAL MEANING AT THE SEMANTICS-PRAGMATICS INTERFACE

ABSTRACT: The present article discusses the notion of conventional meaning at the semantics-pragmatics interface. Such concept is usually the starting point of a traditionally received view on how post-Gricean linguistics should draw the distinction between what is said and what is implicated. In this paper, such line of thought is brought into discussion and the usefulness of the notion is questioned. The article is divided in two parts. In the first part, I defend the idea that conventionality does not capture the interface properties that its advocates intend it to. The second part argues that accepting or rejecting conventionality as part of the set of criteria to assess an adequate theory of meaning partially depends on considering our intuitions about semantic content and pragmatic enrichment.

KEY WORDS: interface; semantics; pragmatics; conventional meaning; intuition.

SUMMARY: 1 . Introduction. 2 . Meaning interfaces. 2.1. The semantics-pragmatics interface (SPI). 3. The distinction between conventional and non-conventional meaning at the SPI. 3.1. The problem of (non) conventional meaning at the SPI. 4. Intuitions versus conventionality. 4.1. The role of intuitions at the interface. 5. Conclusions.

\section{LA NOTION DE SIGNIFIE CONVENTIONNEL DANS L'INTERFACE SEMANTIQUE- PRAGMATIQUE}

RÉSUMÉ: Dans cet article, on discute le concept de sens conventionnel dans l'interface sémantique-pragmatique. Cette notion est le point de départ pour établir la distinction gricéenne entre ce qui a été dit par un locuteur et le contenu implicite ou le contenu communiqué. Dans ce travail, on critique cette ligne, en remettant en question l'utilité de ce concept. La discussion est divisée en deux parties. La première partie fait valoir que la notion du conventionnel ne tient pas compte adéquatement des propriétés d'interface qui sont destinées à décrire et à expliquer ceux qui l'adoptent. Dans la deuxième partie, on envisage que l'acceptation ou le rejet de la conventionalité comme critère d'analyse du sens dépend partiellement du fait que nous puissions accepter ou non que les intuitions sur le contenu sémantique et l'enrichissement pragmatique des expressions linguistiques doivent être prises en compte dans une théorie complète et adéquate du sens.

MOTS CLÉS: interface; sémantique; pragmatique; sens conventionnel; intuition.

SOMMAIRE: 1 . Introduction. 2 Les interfaces du sens. 2.1. L'interface sémantiquepragmatique. 3. Différence entre sens conventionnel et non conventionnel dans l'ISP. 3.1. Le problème du (non) conventionnel dans l'ISP. 4. Intuitions versus conventionnalité. 4.1. Le rôle des intuitions dans l'interface. 5. Conclusions. 


\section{La noción de significado convencional en la interfaz se- mántica-pragmática ${ }^{1}$}

\section{EDUARDO DOMINICCINI}

\section{INTRODUCCIÓN}

Los estudios sobre el significado en las lenguas naturales se han diversificado y especializado tanto en las últimas décadas que resulta difícil controvertir el estatus de la semántica y la pragmática como componentes de una teoria lingüística integral. Sin embargo, la relación misma entre estas dos disciplinas sigue siendo objeto de debate hasta nuestros dias. El problema general es la delimitación del espacio de significado en las lenguas naturales. En cuanto al espacio semántico, en él se ubican, con relativa comodidad, propiedades y relaciones léxicas básicas (polisemia, vaguedad, inclusión, oposición, etc.), mientras que en el espacio pragmático se encuentran los significados que se recuperan a partir del uso en contextos de comunicación. Extendiendo la metáfora, esta división tradicional del espacio de significado representa las regiones adyacentes al área que define la interfaz semántica-pragmática, cuya ubicación limítrofe es el hogar de un gran número de fenómenos que involucran diversos aspectos del contenido codificado y comunicado.

La división de la labor lingüística en el plano del contenido se ha debatido ampliamente en la literatura especializada con base en oposiciones teóricas y empíricas relacionadas con una amplia gama de propiedades del significado. Por ejemplo, de acuerdo con McNally (2013), esta división se ha basado en oposiciones como las que hay entre la denotación y el uso, lo dicho versus lo implicado, la dependencia y la independencia del contexto, y el contenido veritativo-condicional frente al no veritativo-condicional. A estas oposiciones podemos agregar otras relacionadas, como la distinción entre el contenido literal y el no literal (Carston, 2012) y la clásica distinción entre significado convencional y no convencional. Quizás esta última es la más transversal de todas, en el sentido de que está presente de una u otra forma en la mayoría de discusiones sobre la interfaz, sea como parte de un argumento presupuesto o uno explícitamente adoptado.

En el presente trabajo se discuten dos aspectos acerca de la interfaz semántica-pragmática en relación con la noción tradicional de lo convencional que ha dominado la literatura en semántica y pragmática. El primer aspecto tiene que ver con que dicha noción, tal y como se ha utilizado, no es ade-

\footnotetext{
${ }^{1}$ Parte de este trabajo fue presentado en el XV Congreso de la Sociedad Argentina de Lingüística, que tuvo lugar en mayo de 2016 en la Universidad Nacional del Sur. La investigación correspondiente fue financiada por el Instituto Caro y Cuervo.
} 
cuada para explicar fenómenos de la interfaz, por cuanto no existe una correspondencia unívoca entre semántica y convencionalidad, por un lado, y pragmática y no convencionalidad, por otro. El segundo aspecto apunta a que la dicotomía entre lo convencional y lo no convencional es incompatible con una perspectiva de investigación basada en nuestro conocimiento del significado. En la medida en que esta metodología sea considerada válida, el concepto de lo convencional debe ser revisado para determinar si una teoría integral del significado lingüístico puede incluir esa distinción como criterio para determinar propiedades semánticas y pragmáticas de las expresiones lingüísticas.

El trabajo está organizado de la siguiente manera. En la sección 2, se recopilan las ideas fundamentales del concepto de interfaz, haciendo énfasis en los antecedentes de la interfaz semántica-pragmática. La sección 3 presenta y discute el problema teórico de la noción de lo convencional como base de la distinción entre significados dentro de la interfaz. En la sección 4 , se trata el problema metodológico de relacionar el concepto de significado convencional con las intuiciones en la investigación semántica y pragmática. Finalmente, la sección 5 presenta algunas conclusiones y lineamientos para la discusión posterior.

\section{LAS INTERFACES DEL SIGNIFICADO}

Gran parte de los esfuerzos de la lingüistica de las últimas décadas se ha dedicado a las interfaces. Una manera de entender una interfaz lingüística puede ser desde una perspectiva empírica, en la que la interfaz sería un mecanismo de transmisión de información entre distintos módulos, particularmente en el marco de una teoría modular del lenguaje. Así concebida, una idea básica de la interfaz es que un sistema cognitivo complejo debe asegurar las operaciones entre los diferentes subsistemas o módulos; tales operaciones se entienden como intercambios de información, de manera que, en este sentido, las interfaces se postulan como espacios de interacción (Schreiner, 2014).

Existe otra forma de entender una interfaz y es de naturaleza teórica. Por ejemplo, Hackl (2013) explica que la interfaz sintaxis-semántica “[...]tiene que ver con aquellos aspectos de la estructura sintáctica que tienen un efecto en la composición del significado y aquellos aspectos del significado que tienen efectos sistemáticos en la estructura sintáctica" (p. 65). De acuerdo con esta idea, es posible decir que las interfaces son espacios de análisis donde se estudian conjuntos de fenómenos lingüísticos cuya descripción y explicación involucran herramientas provenientes de distintas subdisciplinas, dependiendo del conjunto en cuestión. Por supuesto, esta no es una definición sino una descripción general, ya que establecer con precisión qué hace parte y qué queda excluido de una interfaz no es una tarea fácil. En el caso de la semántica, las interfaces más relevantes se dan 
con la sintaxis y con la pragmática. Para la presente discusión, solo se tendrá en cuenta este sentido teórico de la noción de interfaz, y solo se hará referencia a problemas empíricos sin que esto implique un compromiso con la perspectiva modular mencionada.

Las interfaces en las que se involucra la semántica se originan en diferentes momentos de la lingüística del siglo $\mathrm{XX}$, pero solo en las últimas décadas se han consolidado como áreas de estudio especializadas. Una característica de las interfaces es que se definen más por los conjuntos de fenómenos que estudian que por sus orientaciones teóricas o metodológicas. Por ejemplo, la interfaz entre semántica y sintaxis se ocupa de fenómenos en los que convergen la influencia de la estructuración sintáctica y la de la interpretación semántica, pero las aproximaciones a estos fenómenos no son exclusivamente formales. Algunos temas propios de esta interfaz son el tratamiento de los cuantificadores en la lengua natural, la estructura argumental y la predicación, y el ligamento (binding) de pronombres (Jacobson, 2014). En el caso de la interfaz que relaciona la semántica con la pragmática, uno de los problemas centrales en la investigación de las últimas décadas ha sido el de la distinción entre el contenido semántico de las expresiones lingüisticas y el contenido no explícitamente codificado, pero efectivamente expresado en contextos de comunicación. Esta interfaz agrupa discusiones relacionadas con la determinación del significado en sus dimensiones denotativas, convencionales, de condiciones de verdad y literales, frente a los aspectos de uso, no convencionales, no veritativo-condicionales y no literales que pueden expresarse mediante los enunciados (Jaszczolt, 2012; McNally, 2013). En la sección 2.1 se profundiza la descripción de esta interfaz.

En cuanto a la interfaz sintaxis-semántica-pragmática, uno de los referentes más conocidos es el trabajo de Van Valin en el marco de la Gramática del Papel y la Referencia (GPR). Según el mismo Van Valin (2005, 2008, 2014), la interfaz sintaxis-semántica-pragmática es el objeto de investigación de la GPR. En concreto, la GPR postula que la representación lingüística se basa en tres componentes: uno de representación de significados de expresiones lingüísticas, uno de representación de estructuras sintácticas de las lenguas, y uno de representación de la estructura informativa del discurso (González Vergara, 2006). El enfoque funcionalista de esta propuesta parece contrastar con muchas de las aproximaciones formales de la disciplina, pero la comparación no es definitiva, ya que este modelo pretende abarcar mucho más que las interfaces vistas de manera independiente, además de ser un poco más cercano a los enfoques formales que otros modelos funcionalistas.

Si bien tratar de delimitar el área de interfaz entre dos o más subdisciplinas no deja de ser un problema, como se indicaba antes, es preciso comenzar por una caracterización teórica de la interfaz. Dicha caracterización debe tener en cuenta qué se entiende por cada uno de los componentes que la interfaz relaciona, de manera que se pueda establecer el rango de fenómenos 
que se consideran dentro de ella. Para el caso de la interfaz semántica-pragmática, es posible adoptar parcialmente la misma distinción que se emplea en los estudios pragmáticos en general. Autores como Haugh (2008) contrastan una perspectiva cognitiva-filosófica (anglosajona) con una perspectiva socio-cultural interaccional (europea continental). Así, los problemas específicos que se abordan en cada perspectiva suelen ser de naturaleza distinta. De manera general, en la primera perspectiva se adopta una postura más lingüística que en la segunda, en tanto que el interés se centra más en el significado de las expresiones lingüísticas y los procesos de inferencia que en las prácticas de comportamiento de los hablantes y sus causas o consecuencias sociales y culturales. A las perspectivas mencionadas cabría añadir los enfoques formales, que tanto en semántica como en pragmática han dedicado enormes esfuerzos al desarrollo del campo de estudio de la interfaz. Gran parte de la literatura sobre las propiedades del significado se basan en el estudio de las presuposiciones y las implicaturas por parte de semantistas y pragmatistas de corte formal.

\subsection{LA INTERFAZ SEMÁNTICA-PRAGMÁTICA (ISP)}

Hace más de ochenta años, el famoso lingüista Leonard Bloomfield afirmaba que: "la declaración del significado es [...] el punto débil del estudio del lenguaje y permanecerá igual hasta que el conocimiento humano avance mucho más allá de su estado presente" (Bloomfield, 1933: 140). Está claro que durante esas ocho décadas las disciplinas encargadas de analizar problemas de significado han logrado avances tan significativos que no diriamos que son el punto débil de la lingüística. Estas disciplinas, la semántica y la pragmática, han podido establecer áreas de acción y bases metodológicas adecuadas para trabajar en aspectos bien diferenciados del contenido que es posible comunicar a través de las expresiones lingüísticas (Allan \& Jaszczolt, 2012; Riemer, 2015). Las relaciones entre ambas, sin embargo, han sido problemáticas y han dado origen a todo un campo de estudio que se conoce como la interfaz semántica-pragmática (en adelante ISP). Los temas básicos de la ISP se suelen definir como oposiciones o distinciones con respecto a una gama de aspectos que caracterizan al significado en las lenguas naturales. En general, los debates que se plantean en la ISP son parte de las cuestiones que dieron como resultado la aparición de la pragmática lingüística, pero siguen siendo discutidos con respecto a la semántica en virtud de la innegable relación que existe entre ambas.

Siguiendo a McNally (2013), entre las formas de ver la división de la labor lingüística en el plano del significado, es posible identificar varias distinciones fundamentales, aunque no siempre bien delimitadas. Entre ellas tenemos la distinción entre la denotación de las expresiones lingüísticas y su uso, lo dicho versus lo implicado, la dependencia o independencia del contexto, y el significado veritativo-condicional frente al no veritativo-condicional. En cuanto a la denotación, se trata de una propiedad semántica que se 
establece entre una expresión lingüística y su extensión, es decir, el conjunto de cosas a las que alude dicha expresión (v. gr., la denotación de la expresión "árbol" es la relación entre dicha unidad léxica y la clase de cosas en el mundo que son árboles). El significado denotativo, por tanto, es el que se asocia con lo que cada expresión designa en el sentido más básico. Por su parte, el uso de las expresiones lingüísticas ha sido el concepto sobre el que se ha edificado la tradición pragmática. Las cuestiones sobre el uso configuran una dimensión de análisis que el carácter abstracto de la semántica no cubre. Así, por ejemplo, al analizar el uso concreto de la lengua en situaciones particulares se deben tener en cuenta aspectos como el significado connotativo (opuesto o adicional al denotativo), las variaciones prosódicas, las relaciones sociales y comunicativas, entre otros. En el ámbito de la ISP, la distinción entre denotación y uso implica el análisis de fenómenos de significado que surgen debido a la distancia que hay entre el significado literal y los posibles significados que puede transmitir una expresión cuando se usa en situaciones comunicativas concretas. Los temas pueden abarcar aspectos de semántica y pragmática léxica, estudios prosódicos, retórica, análisis (crítico) del discurso, y pragmática intercultural (McNally, 2013).

Otra distinción clásica en la literatura de la ISP es la que se da entre lo dicho y lo implicado. El referente principal al respecto es el filósofo inglés Herbert Paul Grice. Su contribución al pensamiento moderno ha influenciado tanto la semántica de corte filosófico como la pragmática lingüística. De acuerdo con uno de sus postulados clásicos, el significado que transmite un hablante al expresar algo puede dividirse entre lo que el hablante dice y lo que dar a entender. En muchos de los ejemplos al respecto, es fácil notar que una expresión transmite un contenido informativo básico, pero también un contenido implicado (no dicho) que se recupera en la comunicación (La pregunta “¿Puedes pasarme la sal?" usualmente no pide información sobre una capacidad, sino que solicita que se realice una acción). En esta perspectiva, el dominio de la semántica sería lo dicho (significado de la oración) y el de la pragmática sería lo implicado (significado del enunciado). En términos de la ISP, algunos de los problemas centrales son determinar en qué medida es necesaria la semántica para la interpretación pragmática y qué tipos de contenidos presupuestos o implicados podemos distinguir.

Otra gran pregunta del estudio del significado es en qué medida este depende del contexto. La noción de contexto es un tema ampliamente discutido, pero basta por ahora con distinguir un contexto lingüístico y uno extralingüístico. El primero abarcaría la expresión compleja en la que se ubica un elemento en el discurso, mientras que el segundo comprende una serie de aspectos situacionales (espacio-temporales), cognitivos y sociales de los que diversos enfoques pragmáticos se han apropiado. En el terreno de la ISP, algunos de los problemas estudiados tienen que ver con la ambigüedad en distintos niveles. Por ejemplo, como sostiene McNally (2013), la referencia de los términos de color en las lenguas naturales suele ser ambigua dependiendo de los contextos de uso. En efecto, no parece ser lo mismo el rojo de 
una manzana y el rojo de un rostro ruborizado y, sin embargo, de algún modo, ambos son rojos. La discusión enfrenta a defensores y detractores de las perspectivas minimalista y contextualista con respecto al significado (véase Borg, 2007, 2012). En la ISP, el problema de definir qué es dependiente del contexto y qué es independiente de él se suele asociar con el de la distinción entre el contenido literal y el no literal (y las condiciones de verdad asociadas a ellos) y con la preocupación por separar el conocimiento lingüístico del conocimiento enciclopédico o del mundo.

La distinción entre semántica y pragmática también se ha presentado en términos del significado veritativo-condicional. Este es definido en semántica como el tipo de contenido que afecta las condiciones bajo las cuales una expresión puede ser verdadera (Birner, 2013). Por ejemplo, consideremos el caso en (1), tomado de Birner (2013):

\section{(1) Juan es un verdadero genio.}

Las condiciones de verdad de (1), es decir, las condiciones bajo las cuales (1) puede ser verdadera, tienen que ver con la posibilidad de que efectivamente Juan sea una persona muy inteligente. No obstante, es perfectamente posible usar (1) irónicamente, es decir, con la intención de dar a entender justamente lo opuesto, a saber, que Juan no es muy inteligente. El primer significado sería el que expresa su contenido veritativo-condicional, mientras que el segundo sería el no veritativo-condicional. En la medida en que el contenido veritativo-condicional es invariable, este significado es el que se entiende como independiente del contexto. Dadas las oposiciones clásicas en la disciplina, este significado invariable se suele entender como el contenido semántico, mientras que el que no depende del contexto es el pragmático. Al respecto, McNally (2013) añade que es posible pensar en el contexto lingüístico como un factor que afecta la interpretación cuando varias expresiones con el mismo valor en términos de condiciones de verdad son incompatibles en términos de adecuación o condiciones de felicidad (felicity conditions).

Como puede apreciarse, el espacio del significado en las lenguas naturales se ha dividido de maneras complejas y no siempre bien delimitadas. Cada división muestra una faceta diferente de la interfaz, y esto ha dado como resultado una extensa actividad investigadora dentro de la lingüística moderna. En el mismo sentido, la distinción entre significado convencional y significado no convencional se encuentra ampliamente extendida en la bibliografia de la interfaz. Sin embargo, no es frecuente encontrar reflexiones sobre ella desde una perspectiva metateórica, de modo que se ha dado por sentado como un asunto incontrovertible. En la siguiente sección, se propone una discusión que reta esta postura tradicional. 


\section{LA DISTINCIÓN ENTRE SIGNIFICADO CONVENCIONAL Y NO CONVENCIONAL EN LA ISP}

La noción de lo convencional tiene una larga tradición y una variada aplicación en los estudios del lenguaje. En el sentido más general, un fenómeno es convencional en tanto que se trata de algo aceptado por un grupo y por razones no naturales. En un sentido más restringido y con respecto a la lengua, lo convencional tiene que ver con que muchas expresiones lingüísticas no guardan relación directa con lo que representan. Esta idea se manifiesta con diferentes matices en las obras de Ferdinand de Saussure y Herbert Paul Grice. En el marco de la lingüística estructural de Saussure, es un lugar común la propuesta de que la lengua es un sistema constituido por una gran cantidad de unidades ${ }^{2}$ cuya relación con los significados a los que aluden es convencional (las onomatopeyas y las expresiones del iconismo sonoro son ejemplos de lo opuesto). Según Saussure, “[...] todo medio de expresión recibido de una sociedad se apoya en principio en un hábito colectivo o, lo que viene a ser lo mismo, en la convención" (Grice, 1945: 94). Un primer problema que resulta evidente es que estamos ante distintos tipos de convencionalidad. Por un lado, hablamos de la relación convencional entre el significante y el significado (en las lenguas orales); por otro, hablamos de prácticas comunicativas que se han convencionalizado como normas tácitas de uso por parte de la comunidad lingüística (como los saludos o algunos actos de habla indirectos). Este punto es importante porque, como se verá más adelante, nos plantea la pregunta de qué tipo de convencionalidad es la que la diferencia una interpretación en la semántica de una en la pragmática.

Otro momento en el que podemos identificar una noción lingüística de convencionalidad se encuentra en la propuesta de Grice (1975), específicamente en su famosa distinción entre lo dicho y lo implicado. El filósofo inglés usa la idea de lo convencional del significado de las palabras para sostener que, en determinados casos, existe una brecha entre lo que alguien quiere decir con una expresión lingüística y lo que puede dar a entender usando dicha expresión. Grice parecía dejar clara su manera de entender lo dicho

\footnotetext{
2 El propósito de citar la convencionalidad en la obra de Saussure no es profundizar sobre su perspectiva, sino señalar una propiedad del signo lingüístico. En otras palabras, no pretendo cuestionar la arbitrariedad del signo, sino la convencionalidad como base de una distinción del contenido. Menciono esto en relación con un comentario de uno de los evaluadores anónimos, que señala que la arbitrariedad para Saussure es una propiedad de todo signo de la lengua, de modo que ni siquiera las onomatopeyas son ejemplos adecuados de signos no arbitrarios. Esta es una idea bastante común, pero no es una postura fácil de defender. Cuando Saussure habla del Principio I, la Naturaleza Arbitraria del Signo, se refiere a las onomatopeyas y a las interjecciones como posibles contraejemplos a dicho principio, pero las descarta por su número limitado y por sus orígenes debatibles (Saussure, 1945: 93-95). De acuerdo con Holdcroft (1991), Saussure no presenta una buena defensa de estos puntos. Además, la abundante literatura sobre iconismo sonoro y lenguas de señas refleja el desacuerdo que existe con respecto a las nociones de convencionalidad y arbitrariedad.
} 
al señalar que, al emplear el verbo 'decir', tenía en mente el significado convencional de las palabras emitidas por un hablante (Grice, 1975: 44). Basándonos en este único fragmento, podriamos afirmar que para Grice lo convencional está limitada al nivel del contenido codificado en las unidades lingüísticas. Sin embargo, Grice va más allá y complica la distinción entre "decir" e "implicar" postulando que algunas implicaturas no son conversacionales sino convencionales. Más adelante se volverá sobre este punto. Por ahora, es suficiente establecer que el sentido de lo convencional en Saussure y Grice se puede ver como una conexión no natural entre formas y significados, ya sea que se entienda como una propiedad en algunos tipos de sistemas o como el producto de los hábitos que los hablantes hemos "convenido" en adoptar.

La convencionalidad del significado le ha servido a la pragmática moderna para edificar parcialmente su aparato teórico, de modo que lo convencional y lo no convencional como base de la separación entre semántica y pragmática es una idea recurrente. Por ejemplo, Huang (2007) citando a Katz (1977) recuerda la dicotomía convencional/no convencional en el contexto de las inferencias: "[...] mientras una interpretación semántica, siendo convencional por naturaleza, no puede cancelarse, una inferencia pragmática, que tiene carácter no convencional, sí puede." (Huang, 2007: 213). La cita continúa con un inmediato rechazo a dicha oposición: "Pero como señaló Bach (1999a, 2004), entre otros, esta manera de invocar la división entre semántica y pragmática se tropieza con el hecho problemático de que hay expresiones lingüísticas cuyo significado convencional está fuertemente asociado con el uso" (Huang, 2007: 213). Un ejemplo de las expresiones a las que se refiere Bach es el de las unidades deícticas discursivas, como "a propósito" o "en conclusión”. Según Bach (citado por Huang, 2007), para comprender el significado de estas expresiones se deben especificar sus condiciones de uso (referencia anafórica discursiva). Estos casos muestran que existen expresiones lingüísticas cuya contribución semántica se relaciona directamente con el contexto. El término "contexto" en este caso se refiere al discurso mismo (contexto lingüístico), pero sigue conservando su relación con el uso más que con un tipo de significado convencional. Nótese que no se hace ninguna diferencia entre significado léxico y significado gramatical o funcional, la cual puede relacionarse con la noción de convencionalidad en términos de los distintos grados de fijación del significado de estas unidades. Quizás en esta distinción se aprecia más la utilidad de la noción de lo convencional, pero debería matizarse con cuidado. De cualquier modo, lo importante es tener en cuenta que lo convencional como base para distinguir entre semántica y pragmática es un problema para los casos de significado contextual.

Para analizar con más detalle la situación descrita, retomamos la propuesta de Grice de distinguir entre implicaturas convencionales e implicaturas no convencionales (las conversacionales). De entrada, esta distinción parece muy inusual si recordamos que toda implicatura, por definición, es 
una inferencia a partir de lo dicho, pero no explícita en lo dicho. El clásico ejemplo es el de (2):

(2) Juan es pobre pero honrado.

La idea es que el significado de "pero" expresa un tipo de contraste. E1 contenido implicado sería recuperado a partir de la creencia de que si alguien es pobre entonces no es honrado. Con el contraste se expresa una aclaración que contradice dicha creencia. La razón por la cual Grice usó el término "convencional" para implicaturas como las que se generan en (2) es que ellas se derivan del significado de una palabra o frase cuya contribución semántica se considera convencional, en el sentido de 'significado compartido por los hablantes de una lengua'. Dejando de lado los problemas de la estabilidad de lo convencional en una comunidad lingüística, surge el desafio de ubicar las implicaturas convencionales dentro de la oposición de lo dicho versus lo implicado ${ }^{3}$. Este asunto se trata a continuación.

\subsection{El PROBLEMA DE LO (NO) CONVENCIONAL EN LA ISP}

A pesar de no ser una idea novedosa, problematizar la noción de lo convencional con respecto a la distinción entre semántica y pragmática no ha tenido la repercusión que podría esperarse. Muchos trabajos en pragmática, sean generales o especializados, siguen caracterizando los tipos de significado siguiendo tal distinción. Esta situación es mucho más clara en el ámbito de la interfaz, ya que en ella es fundamental usar las distinciones entre tipos de significado para describir los limites entre los dos ámbitos, y en la mayoría de propuestas la noción de lo convencional parece estar siempre latente. La razón por la cual la noción es problemática es que, aunque pueda tener un cierto valor descriptivo dentro de una caracterización formal del sistema lingüístico, resulta inadecuada cuando se usa para distinguir contenidos semánticos de contenidos pragmáticos. Como se mencionó antes, ni el significado en la dimensión léxica/composicional es completamente convencional, ni el significado en la pragmática es enteramente no convencional. Para explicar el punto, considérense las expresiones en (3) y (4):

(3) A: María compró una casa muy costosa.

B: Claro, ella tiene dinero.

(4) A: ¿Puedes decirme la hora?

B: Son las cuatro en punto.

\footnotetext{
${ }^{3}$ No en vano Horn (2012) la describe como "desempeñando el papel de la fea hermanastra". Para más detalles sobre este tipo de implicaturas, véase la crítica de Bach (1999) y la defensa de Potts (2005).
} 
Para el caso de (3), lo que A dice (en el sentido de Grice) no parece activar directamente inferencias pragmáticas, y sin embargo no diríamos que el significado de la expresión en A es puramente convencional: en este caso, "tener dinero" se suele interpretar como "tener mucho dinero". Este fenómeno puede entenderse de varias maneras. Una de ellas es que se trata de un significado de cuantificación que se ha gramaticalizado en una unidad léxica. De ser (únicamente) así, tal vez podamos hablar de un significado convencional. Otra forma de entender este fenómeno es la que adoptan Bach (1994) con su noción de "implicitura", o los teóricos de la Relevancia como Sperber \& Wilson (2004) y Carston (2002) con la explicatura. Otra opción es plantear que en 3B hay un constituyente no articulado (Huang, 2018). Los pormenores de estos conceptos y sus diferencias quedan fuera del alcance de este trabajo, pero es suficiente mostrar que, en todo caso, el ejemplo cae dentro del conjunto de fenómenos de interfaz en tanto que se trata de un significado comunicado, aunque no explicitamente codificado.

En cuanto a la expresión en (4), tenemos un acto de habla indirecto que, como es bien sabido, se interpreta como una implicatura codificada en forma de petición o con fuerza directiva ("Dime la hora"). Este tipo de expresiones se ha fijado en el uso, llegando hasta tal punto que la manera indirecta de solicitar la información deseada solo se hace evidente cuando se señala en situaciones vinculadas con el humor o cuando debido a ciertos trastornos pragmáticos el hablante contesta la petición directa ("Sí, puedo decirte la hora"). No parece ser el caso que el verbo "poder" esté sufriendo algún tipo de cambio semántico, es decir, no parece tratarse de un caso de polisemia. Sí parece, en cambio, que una estructura del tipo "podrías/puedes $p$ " (donde $p$ es una proposición con algún tipo de fuerza directiva) pasa por un proceso de "pragmaticalización", que como explica Leech, se da por efectos del uso: "a medida que la interpretación convencional aumenta en frecuencia e idiomaticidad, el camino inferencial sufre un corto circuito y se debilita y, eventualmente, se deteriora" (Leech, 2014: 76). En este caso, "convencional" está relacionado con una transición gradual de lo semántico a lo pragmático que se asemeja al proceso de gramaticalización, que es un paso de lo léxico a lo gramatical (o de [-gramatical] a [+gramatical]).

Resumiendo, la noción de convencionalidad en casos como (3) parece quedar indeterminada en el significado expresado como cantidad. Y en casos como (4), es posible decir que se ha convencionalizado una respuesta informativa completa y no se espera una respuesta sobre la capacidad de hacer algo. Los ejemplos sirven para establecer que la distinción entre las expresiones convencionales y las no convencionales no es una tarea completamente transparente. Al respecto, podría postularse que la solución es entender la convencionalidad no como una característica binaria (presente o ausente) sino como una especie de escala o continuo en el que el significado oscila entre lo menos convencional y lo más convencional (como en 4). En otras palabras, se trata de decir que la convencionalidad es un tema de 
grado y no de "todo o nada", como lo hace Börjesson (2014). Para esta autora, que cuestiona lo convencional como base de la distinción entre significado literal y no literal, debemos preguntarnos qué propiedad se supone que recoge la noción de convencionalidad: "¿es el significado convencional de una expresión aquello que está codificado en el léxico o es el significado convencional la interpretación con la que cierta expresión simple o compleja se usa con frecuencia, sin tener en cuenta si el significado se deriva pragmáticamente o si se codifica en el léxico?” (Börjesson, 2014: 35). El argumento de Börjesson para responder la pregunta se fundamenta en datos empíricos acerca del procesamiento de metáforas. Los experimentos citados por la autora sugieren que el contenido idiomático de ciertos modismos muy familiares es procesado de manera muy similar al contenido no idiomático de los significados literales. Esto tendría como consecuencia que la distinción entre significado literal y no literal no seria tan tajante como se ha sostenido. De ser correctos, los resultados mencionados por Börjesson apoyarían la tesis de que el significado metafórico podría ser tan convencional como el literal, y que esta noción debe entenderse como una cuestión de grado. Para reforzar esta idea, la autora cita una escala de grados de convencionalidad propuesta por Busse (1991), que oscila entre casos de convenciones del uso del lenguaje (implicaturas conversacionales particularizadas) y convenciones del lenguaje (casos de significado literal). Nótese que, además de apoyarse en la idea del continuo, Börjesson también acude a una distinción entre tipos de convencionalidad. El resultado es que ahora no solo se trata de aceptar que lo convencional y lo no convencional son extremos de una escala en la que se pueden ubicar las expresiones lingüisticas, sino que también debemos considerar una dimensión adicional, la del significado versus el uso.

$\mathrm{El}$ argumento de Börjesson es interesante y su conclusión parece válida. En efecto, podemos hablar de convencionalidad en diferentes grados y en diferentes ámbitos. Sin embargo, esta nueva forma de ver lo convencional también resulta problemática en el sentido de que le quita poder explicativo a una noción que se supone lo debe tener. El problema surge al tener lo que parecen ser dos variedades de lo convencional. La primera variedad distingue entre la convencionalidad semántica (la relación entre las formas lingüísticas y los significados entendidos como asociaciones conceptuales) y la convencionalidad pragmática (una función de expresiones lingüisticas a prácticas comunicativas ${ }^{4}$ ). La segunda variedad no distingue entre convención semántica y convención pragmática, sino que presenta lo convencional como una característica del significado en general, estableciendo una sola dimensión de lo convencional que se manifiesta como binaria o de grado. En otras palabras, la primera variedad contempla dos tipos de convencionalidad dependiendo del tipo de significado y la segunda adopta un solo tipo

\footnotetext{
${ }^{4}$ Esto debe entenderse en el sentido en el que se interpretan las expresiones formales: una función $F$ que selecciona como argumento una expresión lingüística $l$ y genera como resultado una práctica comunicativa $c$. En notación sería $F(l, c)$, es decir, una función de $l$ a $c$.
} 
que se aplica a toda una gama de significados. La pregunta que plantea Börjesson -qué es el significado convencional de una expresión-, pasa ahora a ser más fundamental: ¿a qué nos referimos con lo convencional? ¿Será el significado, el uso, o ambos? Si la respuesta es que solo el significado en la semántica es convencional y en la pragmática no lo es (lo cual corresponde al uso general del término en la literatura), entonces tenemos una simplificación excesiva de la noción que nos impediría aceptar que las interpretaciones pragmáticas también pueden convencionalizarse. Si la respuesta es que tanto la semántica como la pragmática pueden ser descritas como convencionales, aunque en diferentes sentidos, la noción misma resulta ser poco explicativa: por un lado, es circular en la medida en que el significado se entiende como convencional porque los hablantes han convenido en que ciertas formas correspondan a ciertos significados; por otro lado, nos obliga a aceptar, sin un fundamento sólido, que la convencionalidad es una propiedad atribuible a significados de distinta naturaleza.

La crítica a la noción de lo convencional en lingüistica aquí planteada es más fuerte que las formuladas por los autores citados. Por ejemplo, siguiendo un principio de adecuación, podríamos explicar mejor los datos recurriendo a aspectos no relacionados con lo convencional en ninguno de los sentidos mencionado: la gramaticalización podría ayudar a dar cuenta del significado implicado en (3), y la pragmaticalización explicaría el uso de (4). En resumen, ya sea que se trate de una noción general sobre el significado de las expresiones lingüísticas, o una noción relativa según se atribuya al significado o al uso, la noción misma de lo convencional no parece ser el mejor punto de partida para distinguir entre semántica y pragmática. Dicho de otro modo, las oposiciones entre contenido convencional y no convencional, y entre convencionalidad del sistema y convencionalidad del uso del sistema solo parecen complicar innecesariamente la ISP. Si, por un lado, se trata de una relación excluyente entre dos formas de caracterizar el significado, los casos de semántica no convencional y pragmática convencional hacen insalvable la dicotomía (véase la refutación, a mi juicio convincente, de Börjesson, 2014). Si, por otro lado, se trata de una cuestión de grado, siendo unos casos más convencionales que otros, los limites entre semántica y pragmática se harian aún más difusos producto de definir la interfaz con base en una escala ${ }^{5}$.

Por todo lo anterior, y aunque parezca un reto cuestionar la terminología establecida, la conclusión que podemos sacar es que la dicotomía convencional versus no convencional no resiste un análisis en términos de requisitos de adecuación. En el marco de la ISP, proponemos que el significado puede caracterizarse mejor al tener en cuenta propiedades intrínsecas del contenido a las que tenemos acceso en una perspectiva inferencial. Para tal efecto, la propuesta metodológica supone reivindicar el rol de las intuiciones

\footnotetext{
${ }^{5}$ Por supuesto, es posible descartar por completo la discusión si es que no se acepta la distinción entre semántica y pragmática. Esto es una característica de algunos contextualistas y ocasionalistas con respecto al significado. Al respecto, véase Borg (2012).
} 
como fuente de datos sobre el significado sin de apelar a la noción de convención.

\section{INTUICIONES FRENTE A CONVENCIONALIDAD}

La perspectiva de la interfaz semántica-pragmática que se plantea en este trabajo supone una reflexión acerca de la forma más adecuada de caracterizar la división con respecto al significado lingüístico. En este sentido, hemos visto que la convencionalidad como propiedad del sistema de la lengua, sea absoluta o relativa, no parece ser suficiente para definir la relación entre semántica y pragmática. Las inferencias que se activan en los hablantes en contextos comunicativos, así como las que se usan en el análisis teórico de la ISP, suponen un proceso que no depende de la noción de convencionalidad sino de nuestro conocimiento del significado lingüístico. Por "conocimiento" no me refiero a la dicotomía que se ha establecido con la actuación lingüistica (aunque tampoco la descarto). La referencia se limita a una relación conceptual que tiene que ver con las intuiciones en un sentido epistemológico. Aunque estamos, de nuevo, ante un problema con muchas décadas de historia, sigue siendo un asunto que requiere una mirada distinta de las que han dominado la investigación en la disciplina. El término "intuición" en lingüística está relacionado con los conceptos expresados por términos como "creencias", "juicios", “opiniones”, etc., que usualmente se refieren a un tipo de conocimiento que manifiestan los hablantes acerca de su propia lengua ${ }^{6}$. Para Devitt (2006), las intuiciones son juicios sobre propiedades sintácticas y semánticas de las expresiones lingüísticas. Si a esta noción le añadimos un componente pragmático, tenemos el terreno allanado para tratar de responder a la pregunta de si es posible establecer que las propiedades del significado de las expresiones lingüísticas (sobre su aceptabilidad) pueden caracterizarse apelando a las intuiciones y no a la distinción entre lo convencional y lo no convencional.

¿Por qué nos deberían interesar las intuiciones en el contexto de las discusiones sobre la ISP? Solamente con aceptar que las intuiciones se dan en un nivel representacional y que son metalingüisticas se pueden advertir problemas de diversa índole para la interfaz, como la relación entre contenido semántico y pragmático o entre las expresiones lingüísticas y la accesibilidad de sus propiedades. Además de estos problemas generales, existen discusiones serias acerca del estatus epistemológico y metodológico de las intuiciones dentro del estudio científico del lenguaje y las lenguas. Esto se debe, parcialmente, a que las intuiciones en la interfaz tienen un rol doble. Por un lado, son condiciones que determinan los procesos de inferencia por parte de los hablantes (en el sentido de que nuestro conocimiento del lenguaje nos permite comprender lo que se nos comunica); por otro, pueden

\footnotetext{
${ }^{6}$ En este caso se atiende a un sentido de 'intuición' como el resultado de un proceso de pensamiento, y no a su acepción general de 'facultad de comprender algo sin razonarlo' ni a su acepción coloquial de 'presentimiento'.
} 
ser herramientas útiles para quien investiga propiedades semánticas y pragmáticas.

Los debates sobre las intuiciones en la lingüística son abundantes. Unos están relacionados con los paradigmas de investigación y los conceptos teóricos como la aceptabilidad y la gramaticalidad, y otros tienen que ver con los tipos de datos que se obtienen y los métodos para obtenerlos. Como ejemplo de este segundo tipo, en una nota sobre la metodología en el estudio del nivel pragmático de la lengua, Birner (2013) afirma que las intuiciones personales son inútiles debido a que, como fruto de la introspección, ellas pueden volverse sesgadas hacia la confirmación de nuestras propias hipótesis. Está claro que Birner critica la práctica de contar únicamente con la intuición propia, y que esta autora defiende el uso de métodos que involucren la colaboración de hablantes nativos. Esta postura ejemplifica una tendencia muy fuerte tanto en los estudios del lenguaje como en otras disciplinas. Sin embargo, tiene un problema fundamental que parece simple pero que no ha recibido suficiente atención. Para ejemplificarlo, veamos un caso específico en una discusión sobre sintaxis. Riemer (2009) cuestiona la excesiva dependencia de los juicios intuitivos que muestra la sintaxis generativa, al igual que del rol evidencial que esta les adscribe. El argumento de Riemer descansa en la idea de que muchas de las expresiones que los lingüistas presentan como agramaticales en sus trabajos (mediante el asterisco que precede a una oración) en realidad no lo son. Al respecto, López-Serena (2009) responde que, si un crítico objeta una asignación de agramaticalidad diciendo que esa oración es en realidad aceptable, es porque está usando la intuición propia para llegar a esa conclusión. Sin embargo, aclara la autora, el problema va más allá y tiene que ver con "intuiciones en conflicto", es decir, casos en los que las intuiciones de un autor pueden diferir de las de otro. Esto, asegura López-Serena apoyada en Itkonen (1978), es el resultado de la ya conocida existencia de casos oscuros, pero "no invalidaría el uso de la intuición como la principal fuente de evidencia para la evaluación de la gramaticalidad" (2009, p. 636).

\subsection{EL ROL DE LAS INTUICIONES EN LA INTERFAZ}

Ahora bien, si extrapolamos la discusión al campo de la ISP, podriamos decir que describir y explicar las propiedades del significado exige de quien analiza que apele a sus intuiciones para poder establecer algún tipo de vínculo entre las formas lingüísticas, sus contenidos y sus usos. Por ejemplo, considérese el siguiente intercambio:
A: ¿Podemos salir de paseo el fin de semana?
B: Tengo mucho trabajo. 
Para concluir que la respuesta de B es negativa (una implicatura conversacional), no es necesario (ni suficiente) decir que existen significados convencionales que difieren de lo comunicado. Lo que se necesita es procesar una información y atribuirle una función específica en el contexto dado. Como hablantes, podemos imaginarnos en esa situación y suponer un intercambio exitoso. Como analistas, podemos separar el contenido semántico de la oración de la inferencia a la que da lugar y determinar cómo se ha obtenido la interpretación. En ambos casos, es preciso recurrir a nuestro conocimiento de la lengua, o a lo que en este caso estamos refiriéndonos con "intuiciones" acerca del significado. Así, el hablante usará su intuición para responder apropiadamente en el caso de la conversación y el analista hará lo mismo, en actitud metalingüística, para determinar el tipo de inferencia como fenómeno de ISP. Por supuesto, los detalles del proceso deben ser explicados, para lo cual se puede recurrir a alguna de las opciones teóricas disponibles (la perspectiva neogriceana y la Teoría de la Relevancia son las más destacadas) ${ }^{7}$. En cualquier caso, la intuición sobre el significado es el punto de partida para dar cuenta de la distinción entre la dimensión semántica y la pragmática.

En un principio, nos interesó buscar una explicación de por qué una expresión en particular es o no convencional, de manera que pudiéramos decidir si el concepto mismo de convencionalidad es adecuado para establecer los limites entre semántica y pragmática. Sin embargo, parece más productivo, en términos explicativos, que dicha respuesta evite el camino de la convencionalidad y pase por el de una caracterización adecuada del conocimiento semántico y pragmático. Es preciso aclarar que no se está defendiendo una perspectiva metodológica basada únicamente en intuiciones. Lo que se plantea es que, si las intuiciones son parte tanto del proceso comunicativo como de la actividad analitica investigativa, es difícil ver cómo se podrian excluir por completo de una teoría integral del significado. Al renunciar a la noción de lo convencional y, en su lugar, apelar a las intuiciones, podemos complementar las explicaciones con evidencia empírica, ya que las intuiciones pueden someterse a experimentación. La convencionalidad, por otro lado, aparte de ser distinta para las palabras, las oraciones y los enunciados, no nos dice nada acerca de la relación entre las expresiones y sus significados, ni siquiera porque se trate de una propiedad escalar.

Aunque la diversidad de métodos (lógica, estadística, IRMf, etc.) nos permiten describir algunas propiedades semánticas y pragmáticas, las explicaciones teóricas suponen la necesidad de tener en cuenta las intuiciones, sean las propias o un número significativo de ajenas. Y si nos preguntamos por la validez empírica de nuestra teoria, es preciso recordar que el objeto de estudio no se puede reducir solo a patrones neuronales o a prácticas

\footnotetext{
7 Nuestra posición básicamente comprende integrar un componente pragmático en el principio de composicionalidad clásico de la semántica, de modo que el proceso inferencial se describa en los términos adecuados para la ISP. Esta idea se alinea con las perspectivas que establecen relaciones de interdependencia entre representaciones sintácticas, semánticas y pragmáticas.
} 
sociales. En esa medida, el componente inferencial de la ISP se debe entender también en relación con las intuiciones sobre el significado y el uso.

Esta discusión tiene que ver con las diferencias que enfrentan a lingüistas de corte teórico con lingüistas empíricos. Los últimos están acostumbrados a usar información adquirida en contextos de habla espontánea o dirigida y en bases de datos o corpus de gran tamaño. Dichos estudios se suelen complementar con análisis estadísticos, al tiempo que critican más o menos explícitamente las metodologías que no usan estas herramientas. Una visión conciliadora se puede vislumbrar en trabajos como el de Stolterfoht \& Featherston (2012), cuyo compendio de artículos sobre perspectivas empíricas se fundamenta en la idea de que los distintos métodos para hacer lingüística, en conjunto, pueden ayudar a que nuestras teorias sobre el lenguaje sean cada vez más adecuadas. Sin embargo, el debate específico sobre las intuiciones está lejos de darse por terminado, y las consecuencias teóricas y metodológicas son tan numerosas como variadas para cada parte de la lingüistica, lo cual hace indispensable crear los espacios adecuados para evaluarlas en detalle (para una discusión más amplia al respecto, véase Schütze \& Sprouse, 2013 y Stokhof, 2011).

\section{Conclusiones}

La distinción entre semántica y pragmática ha sido tradicionalmente asociada, entre otras, con la noción de convencionalidad. Esta noción presenta problemas de fondo y aunque varios autores los han señalado, han optado por resolver la cuestión recurriendo a distinciones que limitan o extralimitan el concepto. El asunto va más allá de establecer si se trata de una dicotomía bien delimitada o una cuestión de grados en una escala que va de lo más a lo menos convencional. Ya sea que se tenga una noción distinta de lo convencional para lo semántico y para lo pragmático o que solo haya una noción que se aplica a distintos fenómenos de significado, el valor explicativo de este concepto se deshace una vez que se analizan fenómenos de interfaz.

Mientras que en un flanco de la discusión sobre la ISP se propone eliminar la noción de lo convencional como base de la distinción entre las dos disciplinas, en otro se defiende la idea de que las intuiciones sobre el significado y sobre su uso deben tener un lugar en la teoría. El valor de las intuiciones como datos lingüísticos reside en que pueden ser la base de la confirmación o la refutación de hipótesis tanto en el hablante que está ejerciendo su capacidad metalingüística como en la investigadora que evalúa los juicios de aceptabilidad de otras personas, los suyos propios y las propiedades del significado que pretende analizar. Aclarando que la propuesta no es analizar la semántica y la pragmática exclusivamente con base en intuiciones, se defiende que no es posible rechazarlas por completo sin considerar las consecuencias teóricas y metodológicas de tal maniobra. En la 
medida en que estas ideas se debatan con mayor fuerza y profundidad, lograremos mejorar nuestro aparato teórico para la investigación de los fenómenos de la interfaz semántica-pragmática.

\section{REFERENCIAS}

ALLAN, K. \& JASZCZOLT, K. M. (Eds.). (2012): The Cambridge Handbook of Pragmatics. Cambridge: Cambridge University Press.

BACH, K. (1999): The Myth of Conventional Implicature. Linguistics and Philosophy, 22, pp. 327366.

BACH, K. (1994): Conversational impliciture. Mind and Language, 9, pp. 124-162.

BIRNER, B. J. (2013): Introduction to Pragmatics. Oxford: WileyBlackwell.

BLOOMFIELD, L. (1933): Language. Londres: George Allen \& Unwin.

BORG, E. (2012): Pursuing Meaning. Oxford: Oxford University Press.

BORG, E. (2007): Minimalism versus contextualism in semantics. En Context-sensitivity and Semantic Minimalism: New Essays on Semantics and Pragmatics. Oxford: Oxford University Press, pp. 339-359.

BÖRJESSON, K. (2014): The Semantics-Pragmatics controversy. Berlin/Boston: De Gruyter Mouton.

CARSTON, R. (2012): Metaphor and the literal/nonliteral distinction. En K. Allan \& K. M. Jaszczolt (Eds.), The Cambridge Handbook of Pragmatics, Cambridge: Cambridge University Press, pp. 469-492.

CARSTON, R. (2002): Thoughts and Utterances: The Pragmatics of Explicit Communication. Oxford: Blackwell.

DEVITT, M. (2006): Intuitions in linguistics. The British Journal for the Philosophy of Science, 57, pp. 481-513.

GONZÁLEZ VERGARA, C. (2006): La Gramática del Papel y la Referencia: una aproximación al modelo. Onomázein, 14(2), pp. 101-140.

GRICE, H. P. (1975): Logic and conversation. En P. Cole \& J. Morgan (Eds.), Syntax and Semantics, Volume 3: Speech Acts, New York: Academic Press, pp. 43-58.

HACKL, M. (2013): The syntax-semantics interface. Lingua, 130, pp. 66-87.

HAUGH, M. (2008): Intention in pragmatics. Intercultural Pragmatics, 5(2), pp. 99-110.

HOLDCROFT, D. (1991): Saussure: Signs, system, and arbitrariness. Cambridge: Cambridge University Press.

HORN, L. (2012): Implying and inferring. En K. Allan \& K. M. Jaszczolt (Eds.), The Cambridge Handbook of Pragmatics, Cambridge: Cambridge University Press, pp. 69-86.

HUANG, Y. (2018): Unarticulated constituents and neo-Gricean pragmatics. Language and Linguistics, 19(1), pp. 1-31.

HUANG, Y. (2007): Pragmatics. New York: Oxford University Press.

JACOBSON, P. (2014): Compositional semantics: An introduction to the syntax/semantics interface. Oxford: Oxford University Press.

JASZCZOLT, K. M. (2012): Semantics/pragmatics boundary disputes. En C. Maienborn, K. von Heusinger, \& P. Portner (Eds.), Semantics: An International 
Handbook of Natural Language Meaning, 3, Berlin/Boston: De Gruyter Mouton.

LEECH, G. (2014): The Pragmatics of Politeness. Nueva York: Oxford University Press.

LÓPEZ-SERENA, A. (2009): Intuition, acceptability and grammaticality: a reply to Riemer. Language Sciences, 31, pp. 634-648.

MCNALLY, L. (2013): Semantics and Pragmatics. Wiley Interdisciplinary Reviews: Cognitive Science, 4, pp. 285-297.

POTTS, C. (2005): The Logic of Conventional Implicatures. Oxford: Oxford University Press.

RIEMER, N. (Ed.): (2015): The Routledge Handbook of Semantics. Londres: Routledge.

RIEMER, N. (2009): Grammaticality as evidence and as prediction in a Galilean linguistics. Language Sciences, 31, pp. 612633.

SAUSSURE, F. DE. (1959): Curso de lingüística general. Buenos Aires: Editorial Losada.

SCHREINER, S. (2014): The syntaxsemantics/pragmatics interface. En A. Carnie, Y. Sato, \& D. Siddiqi (Eds.), The Routledge Handbook of Syntax, Londres: Routledge, pp. 307-321.

SCHÜTZE, C. T. \& SPROUSE, J. (2013): Judgment data. En R. J. Podesva \& D. Sharma (Eds.), Research methods in linguistics,
Cambridge: Cambridge University Press, pp. 27-50.

SPERBER, D. \& WILSON, D. (2004): La Teoría de la Relevancia. Revista de Investigación Lingüistica, 7, pp. 237-286.

STOKHOF, M. (2011): Intuitions and competence in formal semantics. The Baltic International Yearbook of Cognition, Logic and Communication, 6, pp. 123.

STOLTERFOHT, B. \& FEATHERSTON, S. (Eds.): (2012): Empirical Approaches to Linguistic Theory. Berlin/Boston: De Gruyter Mouton.

VAN VALIN, R. (2014): Role and Reference Grammar. En A. Carnie, D. Siddiqi, \& Y. Sato (Eds.), Routledge Handbook of Syntax, Londres: Routledge, pp. 579603.

VAN VALIN, R. (2005): Exploring the syntax-semantics interface. Cambridge: Cambridge University Press.

VAN VALIN, R. (Ed.): (2008): Investigations of the Syntax-SemanticsPragmatics Interface. Amsterdam: John Benjamins.

WILLEMS, K. (2012): Intuition, introspection and observation in linguistic inquiry. Language Sciences, 34, pp. 665-681. 